\title{
Shape-phase transitions in mixed parity systems and the onset of octupole deformation
}

\author{
Serdar Kuyucak ${ }^{1}$ \\ Department of Theoretical Physics, Research School of Physical Sciences, \\ Australian National University, Canberra, ACT 0200, Australia
}

\begin{abstract}
Shape-phase transitions in mixed parity boson systems are studied using mean field theory. It is shown that without parity projection, shape transitions in mixed parity systems are very similar to those in positive parity systems, both requiring a finite interaction strength for the onset of deformation. The shape-phase diagram in mixed parity systems, however, changes significantly after parity projection, with the critical point for the onset of octupole deformation moving to zero strength. Shape transitions in the Lipkin model are shown to exhibit the same features, indicating that this is a direct consequence of parity projection in finite, mixed parity systems independent of the nature of interacting particles.
\end{abstract}

PACS: 21.60.Ev; 21.60.Fw

The low-lying negative parity states in even-even collective nuclei are described in terms of the octupole degree of freedom (see [1,2] for recent reviews). Experimental study of the octupole states is hindered by the much stronger quadrupole collectivity which limits their accessibility. Thanks to the advances in detector technology, this situation has changed dramatically in recent years. Exploiting the capabilities of the $\gamma$-ray arrays Eurogam and Gammasphere, the range of negative parity spectra in actinide nuclei $\mathrm{Rn}, \mathrm{Ra}$ and $\mathrm{Th}$ has been greatly extended $[3,4]$. These new data, in turn, provide fresh challenges for collective models, the most interesting one being the description of the conjectured transition from the octupole-vibrational to the octupole-deformed shapes in actinides. In this letter, we present mean field studies of this shapephase transition in two analytically solvable models, the interacting boson model (IBM) [5] and the Lipkin model [6], that will shed further light into this question.

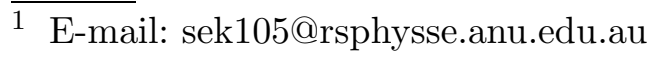

Preprint submitted to Elsevier Preprint

25 December 2018 
We first briefly review the spherical $\rightarrow$ quadrupole-deformed shape transition in the $s d$-IBM [7-9], which provides a useful reference point for the octupole case. A convenient form of the Hamiltonian is

$$
H_{s d}=\varepsilon_{d} \hat{n}_{d}-\kappa_{2} Q \cdot Q
$$

where the one-body term tries to keep the system spherical while the quadrupole interaction drives it towards deformation. Here $\hat{n}_{d}=\sum_{\mu} d_{\mu}^{\dagger} d_{\mu}$ is the $d$-boson number operator and $Q=\left[s^{\dagger} \tilde{d}+d^{\dagger} s\right]+\chi_{d}\left[d^{\dagger} \tilde{d}\right]^{(2)}$ is the quadrupole operator. For simplicity, we use the axially symmetric intrinsic state in the mean field analysis (the full state leads to the same result)

$$
\left|N, \beta_{2}\right\rangle=\left[N !\left(1+\beta_{2}^{2}\right)^{N}\right]^{-1 / 2}\left(s^{\dagger}+\beta_{2} d_{0}^{\dagger}\right)^{N}|0\rangle
$$

Evaluating the expectation value of the Hamiltonian (1) in the intrinsic state (2) yields the energy surface

$$
E\left(\beta_{2}\right)=N^{2} \kappa_{2}\left[\frac{\eta_{2} \beta_{2}^{2}}{1+\beta_{2}^{2}}-\left(\frac{2 \beta_{2}+\bar{\chi}_{d} \beta_{2}^{2}}{1+\beta_{2}^{2}}\right)^{2}\right]
$$

where $\bar{\chi}_{d}=-\sqrt{2 / 7} \chi_{d}$, and $\eta_{2}=\epsilon_{d} / N \kappa_{2}$ provides a convenient parameter to study the shape transition. Here and in the following, the $1 / N$ corrections are neglected in the spirit of the large $N$ assumption inherent in the mean field theory. Variation of $E\left(\beta_{2}\right)$ with respect to $\beta_{2}$ gives

$$
\beta_{2}\left[\eta_{2}\left(1+\beta_{2}^{2}\right)-2\left(2+\bar{\chi}_{d} \beta_{2}\right)\left(1+\bar{\chi}_{d} \beta_{2}-\beta_{2}^{2}\right)\right]=0
$$

Analysis of Eqs. $(3,4)$ shows that the critical point for spherical $\rightarrow$ deformed shape transition is given by $\eta_{2}^{\text {cr }}=4+\bar{\chi}_{d}^{2}$. For $\eta_{2}>\eta_{2}^{\text {cr }}$, the system has a spherical shape with $\beta_{2}=0$, and for $\eta_{2}<\eta_{2}^{\text {cr }}$, it becomes deformed with $\beta_{2}$ determined from Eq. (4). At the critical point, the spherical and deformed solutions coexist with energies $E\left(\beta_{2}=0\right)=E\left(\beta_{2}=\bar{\chi}_{d} / 2\right)=0$. We note that angular momentum projection leads to the same energy expression as (3) to leading order in $N$ [10], hence restoration of the rotational invariance leads to a qualitatively similar shape-phase diagram.

The proper framework for discussion of shape-phase transitions in mixed parity systems is the spdf-IBM which has been shown to give an adequate description of the positive and negative parity spectra in collective nuclei [11-14]. However, the mean field analysis of the spdf-IBM is quite complicated which tends to obscure otherwise a very simple conclusion. To illustrate the main 
ideas involved in a simple case, we first discuss the spherical $\rightarrow$ octupoledeformed shape transition in the $s f$-IBM using the Hamiltonian

$$
H_{s f}=\varepsilon_{f} \hat{n}_{f}-\kappa_{3} O \cdot O
$$

where $\hat{n}_{f}$ is the $f$-boson number and $O=\left[s^{\dagger} \tilde{f}+f^{\dagger} s\right]^{(3)}$ is the octupole operator. This Hamiltonian is similar to (1) when $\chi_{d}=0$, and like the $\gamma$-instability in that case, it is unstable in the direction of deformation. That is, each of the octupole mean fields $\beta_{3 \mu}$ is populated equally, and the energy surface is a function of $\beta_{3}^{2}=\sum_{\mu} \beta_{3 \mu}^{2}$. With this provision, the intrinsic state and the corresponding energy surface (without PP) are given by

$$
\begin{aligned}
\left|N, \beta_{3}\right\rangle & =\left[N !\left(1+\beta_{3}^{2}\right)^{N}\right]^{-1 / 2}\left(s^{\dagger}+\vec{\beta}_{3} \cdot \vec{f}^{\dagger}\right)^{N}|0\rangle, \\
E\left(\beta_{3}\right) & =N^{2} \kappa_{3}\left[\frac{\eta_{3} \beta_{3}^{2}}{1+\beta_{3}^{2}}-\left(\frac{2 \beta_{3}}{1+\beta_{3}^{2}}\right)^{2}\right]
\end{aligned}
$$

where $\eta_{3}=\epsilon_{f} / N \kappa_{3}$. Variation of $E\left(\beta_{3}\right)$ w.r.t. $\beta_{3}$ gives

$$
\beta_{3}\left[\eta_{3}\left(1+\beta_{3}^{2}\right)-4\left(1-\beta_{3}^{2}\right)\right]=0
$$

which has the solutions

1) $\beta_{3}=0, \quad E=0$,

2) $\beta_{3}^{2}=\frac{4-\eta_{3}}{4+\eta_{3}}, \quad E=-\frac{N^{2} \kappa_{3}}{16}\left(\eta_{3}-4\right)^{2}, \quad \eta_{3} \leq 4$.

It is clear from these solutions that for $\eta>4$, the shape is spherical, and the critical point for the onset of octupole deformation occurs at $\eta_{3}^{\text {cr }}=4$. Thus, just like in the quadrupole case, a finite interaction strength, given by $\kappa_{3}=\epsilon_{f} / 4 N$, is required for the shape transition to take place. Note that one can obtain this result without explicit solutions by simply expanding the energy surface around $\beta_{3}$, and looking at the coefficient of $\beta_{3}^{2}$. From Eq. (6), $E\left(\beta_{3}\right) \simeq N^{2} \kappa_{3}\left[\left(\eta_{3}-4\right) \beta_{3}^{2}+\ldots\right]$, and it is obvious from the sign of $\eta_{3}-4$ that the $\beta_{3}=0$ solution is stable for $\eta_{3}>4$ but becomes unstable for $\eta_{3}<4$, leading to a deformed solution. This method of determining the critical point is useful in cases where the extremum conditions cannot be solved explicitly.

The intrinsic state in Eq. (6) has mixed parity, and it is important to see how PP influences the shape transition characteristics discussed above. The projection operator onto good parity is given by

$$
P_{\pi}=(1+\pi \mathcal{P}) / 2
$$


where $\pi= \pm 1$ is the parity quantum number and $\mathcal{P}$ is the parity operator. Under $\mathcal{P}$, boson operators transform as $\mathcal{P} b_{l m}^{\dagger} \mathcal{P}=(-1)^{l} b_{l m}^{\dagger}$, hence $\mathcal{P}$ acting on the intrinsic state (6) gives

$$
\mathcal{P}\left|N, \beta_{3}\right\rangle=\left|N,-\beta_{3}\right\rangle
$$

From Eqs. (9) and (10), the projected states can be written as

$$
P_{\pi}\left|N, \beta_{3}\right\rangle=\left(\left|N, \beta_{3}\right\rangle+\pi\left|N,-\beta_{3}\right\rangle\right) / 2 \text {. }
$$

Binomial expanding the even and odd spin parts in (11), it is easy to see that for $\pi=+1$, it has an even number of $f$ bosons, and for $\pi=-1$, it has an odd number of $f$ bosons. Normalization of the projected state (11) is given by

$$
\mathcal{N}_{\pi}=\left\langle N, \beta_{3}\left|P_{\pi}\right| N, \beta_{3}\right\rangle=\left(1+\pi r^{N}\right) / 2
$$

where

$$
r=\left(1-\beta_{3}^{2}\right) /\left(1+\beta_{3}^{2}\right)
$$

gives a convenient measure of parity mixture in the condensate; for $r=1$ $\left(\beta_{3}=0\right)$, it consists of only $s$ bosons, while for $r=0\left(\beta_{3}=1\right)$, it is an equal admixture of $s$ and $f$ bosons.

The energy surface of the Hamiltonian (5) with PP is given by

$$
\begin{aligned}
E_{\pi}\left(\beta_{3}\right) & =\frac{1}{\mathcal{N}_{\pi}}\left\langle N, \beta_{3}\left|H P_{\pi}\right| N, \beta_{3}\right\rangle, \\
& =\frac{N^{2} \kappa_{3}}{1+\pi r^{N}}\left\{\frac{\eta_{3} \beta_{3}^{2}}{1+\beta_{3}^{2}}\left(1-\pi r^{N-1}\right)-\left(\frac{2 \beta_{3}}{1+\beta_{3}^{2}}\right)^{2}\right\} .
\end{aligned}
$$

Variation of Eq. (14) leads to a complicated equation that cannot be solved analytically. Nevertheless, by plotting the energy surface for various values of $\eta_{3}$, one can gain useful insights into the nature of the shape transition. In Fig. 1 we show plots of Eqs. (6) and (14) for $\eta_{3}=4,40$ and 400. The first value corresponds to the critical case without PP. The next two values represent a 10 - and 100-fold reduction in the octupole strength compared to the first case for a fixed $\epsilon_{f} / N$. In the top figure, the energy surface without PP (dashed line) exhibits the typical flat-bottomed curve expected at the critical point. After PP the energy surface for $\eta_{3}=4$ is seen to become deformed (solid line). What is surprising, however, is that despite the orders of magnitude reduction in the octupole strength in the middle and bottom figures, the $\beta_{3}=0$ solution 
remains unstable and the minimum of $E_{+}$always occurs at $\beta_{3}^{2}>0$, that is, the energy surface after PP remains deformed. As $\kappa_{3}$ is progressively reduced, the well depth and deformation decrease proportionally but the energy surface itself (solid line) stays more or less shape invariant. To find the critical point analytically, we expand $E_{+}(14)$ around $\beta_{3}=0$

$$
E_{+} \simeq N^{2} \kappa_{3}\left[-2 \beta_{3}^{2}+\left(\eta_{3}-2\right) \beta_{3}^{4}+\ldots\right]
$$

It is clear from Eq. (15) that for any $\kappa_{3}>0$, there is a deformed minimum, and hence the critical point is given by $\kappa_{3}=0$. That is, the onset of the octupole deformation is immediate in the presence of any octupole strength, and the spherical phase found in the study of shape transition without PP disappears after PP. This situation is depicted in Fig. 2, where $\beta_{3}$ corresponding to the absolute minimum of the energy surface with and without PP are plotted against $1 / \eta_{3}$. The sharp spherical $\rightarrow$ octupole-deformed transition exemplified by the dashed curve (which is also representative of the quadrupole case) is replaced by a smoothly varying curve after $\mathrm{PP}$, and the critical point moves from $1 / \eta_{3}=0.25$ to 0 (or from $\kappa_{3}=\epsilon_{f} / 4 N$ to 0 ).

To understand this somewhat surprising result, it is worthwhile to look at the effect of PP on individual terms by comparing Eqs. (6) and (15). The leading order $\left(\beta_{3}^{2}\right)$ contribution to the one-body energy is seen to vanish after PP, whereas the octupole term survives, loosing only half of its strength. A more direct way to see the effect of PP is to expand the condensate

$$
\begin{aligned}
\left(s^{\dagger}+\vec{\beta}_{3} \cdot \vec{f}^{\dagger}\right)^{N}= & \left(s^{\dagger}\right)^{N}+N\left(s^{\dagger}\right)^{N-1} \vec{\beta}_{3} \cdot \vec{f}^{\dagger} \\
& +(1 / 2) N(N-1)\left(s^{\dagger}\right)^{N-2}\left(\vec{\beta}_{3} \cdot \vec{f}^{\dagger}\right)^{2}+\ldots,
\end{aligned}
$$

and look at the overlaps of various terms. Denoting the states in the expansion by their $f$ boson number $n_{f}$, the leading contribution to the one-body term comes from the (1-1) matrix element (m.e.) (i.e. $\left.\left\langle 1\left|\hat{n}_{f}\right| 1\right\rangle\right)$, which goes like $\beta_{3}^{2}$. However, after PP, odd $n_{f}$ terms are projected out, so the leading term has to come from the (2-2) m.e., which goes like $\beta_{3}^{4}$. In contrast, the leading (equal) contributions to the octupole term come from the (1-1) and $(0-2+2-0)$ m.e., and PP blocks only the first one, leaving the second one intact. Thus the net effect of PP is to lift the obstruction of the one-body operator against the formation of a deformed system.

The above conclusion on the effect of PP on shape transitions in mixed parity systems can be generalized to arbitrary boson spins, and in particular to the spdf-IBM. However, as stressed before this case is rather complicated and is deferred to a more detailed study. Due to weaker dipole collectivity, the $p$ boson is weakly coupled to the $s d f$ system, and should only have a perturbative 
effect on the $s d f$-IBM results. Thus, for our purposes, it is sufficient to study the physically most relevant case, namely, the onset of octupole deformation in a quadrupole deformed system within the framework of the $s d f$-IBM. The Hamiltonian is given by a combination of Eqs. (1) and (5)

$$
H_{s d f}=\varepsilon_{d} \hat{n}_{d}+\varepsilon_{f} \hat{n}_{f}-\kappa_{2} Q \cdot Q-\kappa_{3} O . O
$$

where the quadrupole and octupole operators are generalized to

$$
\begin{aligned}
& Q=\left[s^{\dagger} \tilde{d}+d^{\dagger} s\right]^{(2)}+\chi_{d}\left[d^{\dagger} \tilde{d}\right]^{(2)}+\chi_{f}\left[f^{\dagger} \tilde{f}\right]^{(2)}, \\
& O=\left[s^{\dagger} \tilde{f}+f^{\dagger} s\right]^{(3)}+\chi_{3}\left[d^{\dagger} \tilde{f}+f^{\dagger} \tilde{d}\right]^{(3)}
\end{aligned}
$$

Numerical studies of this Hamiltonian with a general intrinsic state show that the ground state remains axially symmetric for realistic choices of parameter [15]. Using the intrinsic state

$$
\left|N, \beta_{2}, \beta_{3}\right\rangle=\frac{\left(s^{\dagger}+\beta_{2} d_{0}^{\dagger}+\beta_{3} f_{0}^{\dagger}\right)^{N}}{\left[N !\left(1+\beta_{2}^{2}+\beta_{3}^{2}\right)^{N}\right]^{1 / 2}}|0\rangle,
$$

the energy surface of the Hamiltonian (17) after PP is given by

$$
\begin{aligned}
E_{\pi}\left(\beta_{2}, \beta_{3}\right)= & \frac{N^{2}}{1+\pi r^{N}}\left\{\sum_{l=2}^{3} \frac{\epsilon_{l} \beta_{l}^{2}}{N \beta_{+}^{2}}\left(1+(-1)^{l} \pi r^{N-1}\right)\right. \\
& -\frac{\kappa_{2}}{\beta_{+}^{4}}\left[\left(2 \beta_{2}+\bar{\chi}_{d} \beta_{2}^{2}+\bar{\chi}_{f} \beta_{3}^{2}\right)^{2}+\pi r^{N-2}\left(2 \beta_{2}+\bar{\chi}_{d} \beta_{2}^{2}-\bar{\chi}_{f} \beta_{3}^{2}\right)^{2}\right] \\
& \left.-\frac{\kappa_{3}}{\beta_{+}^{4}}\left(2 \beta_{3}+2 \bar{\chi}_{3} \beta_{2} \beta_{3}\right)^{2}\right\}
\end{aligned}
$$

where $\beta_{+}^{2}=1+\beta_{2}^{2}+\beta_{3}^{2}, \quad r=\left(1+\beta_{2}^{2}-\beta_{3}^{2}\right) / \beta_{+}^{2}, \bar{\chi}_{f}=\sqrt{4 / 21} \chi_{f}$ and $\bar{\chi}_{3}=$ $-\sqrt{4 / 15} \chi_{3}$. The energy surface without PP follows from Eq. (20) by setting $\pi=0$. In the case of no PP, an analysis similar to Eqs. $(7,8)$ can be carried out leading to an octupole vibrational phase $\left(\beta_{3}=0\right.$ and $\beta_{2}$ given by Eq. (4)), and a deformed phase $\left(\beta_{3}>0\right)$ which is too complicated to present here. Nevertheless the critical point can be obtained from the expansion of the energy surface around $\beta_{3}=0$ as

$$
\kappa_{3}=\frac{\epsilon_{f}\left(1+\beta_{2}^{2}\right)+2 N \kappa_{2} \beta_{2}\left(2+\bar{\chi}_{d} \beta_{2}\right)\left(\beta_{2}-\bar{\chi}_{f}\right)}{4 N\left(1+\bar{\chi}_{3} \beta_{2}\right)^{2}} .
$$

The quadrupole deformation is seen to have modified the critical point for the onset of octupole deformation but still a finite $\kappa_{3}$ is required. A similar 
expansion of Eq. (20) for $\pi=+1$ shows that the leading term is proportional to $-\kappa_{3} \beta_{3}^{2}$ as in the spherical case (15). Thus after PP, the critical point again occurs at $\kappa_{3}=0$.

As a final example, we consider shape transitions in the Lipkin model [6], which has been shown to mimic, at the mean field level, the octupole deformation effects obtained from the more realistic Hartree-Fock-Bogolyubov calculations [16]. In the Lipkin model, $N=2 j+1$ nucleons occupy two levels that have the same angular momentum $j$ but opposite parity $\pi$. The Hamiltonian with one- and two-body terms is given by

$$
H=\epsilon K_{0}-\frac{1}{2} \kappa\left(K_{+} K_{+}+K_{-} K_{-}\right)
$$

where $K$ 's are the $\mathrm{SU}(2)$ quasi-spin operators defined as

$$
\begin{aligned}
& K_{0}=\frac{1}{2} \sum_{m}\left(a_{m+}^{\dagger} a_{m+}-a_{m-}^{\dagger} a_{m-}\right), \\
& K_{+}=\sum_{m} a_{m+}^{\dagger} a_{m-}, \quad K_{-}=\left(K_{+}\right)^{\dagger},
\end{aligned}
$$

with the quantum numbers $k=N / 2, \mu=-N / 2,-N / 2+1, \ldots, N / 2$.

In the "spherical" (or harmonic) limit $(\kappa=0)$, the ground state and its energy are given by

$$
\left|\phi_{0}\right\rangle=\prod_{m} a_{m-}^{\dagger}|0\rangle, \quad E_{0}=-\epsilon N / 2 .
$$

The excited states are obtained by repeated application of $K_{+}$on the ground state, which generates a finite, harmonic spectrum, $E_{n}=E_{0}+n \epsilon, n=$ $1, \ldots, N$. For $\kappa>0$, the two body-interaction mixes the $\pi=+1$ and -1 levels, and to find the ground state of the system, one needs to use an intrinsic state with mixed parity

$$
|N, \beta\rangle=\left[\left(1+\beta^{2}\right)^{N}\right]^{-1 / 2} \prod_{m}\left(a_{m-}^{\dagger}+\beta a_{m+}^{\dagger}\right)|0\rangle,
$$

where the mean field $\beta$ describes the "deformation" of the system. With the intrinsic state (25), the energy surface of the Hamiltonian (22) without PP is given by

$$
E(\beta)=-\frac{\epsilon N}{2}+N^{2} \kappa\left[\frac{\eta \beta^{2}}{1+\beta^{2}}-\left(\frac{\beta}{1+\beta^{2}}\right)^{2}\right]
$$


where $\eta=\epsilon / N \kappa$. Apart from the constant term, the energy surface (26) is very similar to that of the $s f$-IBM (6), and it is easy to see from its expansion that the critical point for the onset of deformation occurs at $\eta=1$ or $\kappa=\epsilon / N$.

Parity projection in the Lipkin model is defined just like in the boson case Eqs. (9-11) and yields the following energy surface

$$
E_{\pi}(\beta)=\left[-\frac{\epsilon N r}{2}-N^{2} \kappa\left(\frac{\beta}{1+\beta^{2}}\right)^{2}\right] \frac{1+\pi r^{N-2}}{1+\pi r^{N}},
$$

where $r$ is defined as in Eq. (13). Expanding $E_{+}(\beta)$ around $\beta=0$ gives $E_{+} \simeq-\epsilon N / 2-N^{2} \kappa \beta^{2}+\ldots$ Thus the critical point again moves to $\kappa=0$ after PP.

In conclusion, using analytically solvable models of mixed parity systems we have demonstrated that a sharp transition to octupole deformation, similar to the onset of quadrupole deformation, occurs only if one ignores PP. After PP, the character of the shape transition changes completely, and the vibrational phase disappears. While one can still use the terminology and formalism of octupole vibrations for small $\beta_{3}$, this is only an approximation and not a well defined phase as in quadrupole vibrations. A similar effect of the restoration of discrete symmetries in finite systems is well known in the BCS theory, where the critical pairing strength vanishes after the number projection. The effect of $\mathrm{PP}$ on shape transitions, however, seems to have been ignored in the literature so far. Implications of this result for the octupole states in actinides will be discussed in a longer article.

Parts of this work were carried out while visiting the Japan Atomic Energy Research Institute and the Institute for Nuclear Theory at the University of Washington. Hospitality and support of these Institutes are acknowledged with thanks.

\section{References}

[1] I. Ahmad and P.A. Butler, Ann. Rev. Nucl. Part. Sci. 43 (1993) 71.

[2] P.A. Butler and W. Nazarewicz, Rev. Mod. Phys. 68 (1996) 349.

[3] J.F. Smith et al., Phys. Rev. Lett. 75 (1995) 1050.

[4] J.F.C. Cocks et al., Phys. Rev. Lett. 78 (1997) 292; Nucl. Phys. A 645 (1999) 61.

[5] F. Iachello and A. Arima, The Interacting Boson Model (Cambridge University Press, Cambridge, 1987). 
[6] H.J. Lipkin, N. Meshkov and A.J. Glick, Nucl. Phys. A 62 (1965) 188.

[7] A.E.L. Dieperink and O. Scholten, Nucl. Phys. A 346 (1980) 125.

[8] J.N. Ginocchio and M.W. Kirson, Nucl. Phys. A 350 (1980) 31.

[9] F. Iachello, N.V. Zamfir and R.F. Casten, Phys. Rev. Lett. 81 (1998) 1191.

[10] S. Kuyucak and I. Morrison, Ann. Phys. (NY) 181 (1988) 79.

[11] J. Engel and F. Iachello, Phys. Rev. Lett. 54 (1985) 1126; Nucl. Phys. A 472 (1987) 61.

[12] T. Otsuka, Phys. Lett. B 182 (1986) 256.

[13] T. Otsuka and M. Sugita, Phys. Lett. B 209 (1988) 140.

[14] D. Kusnezov and F. Iachello, Phys. Lett. B 209 (1988) 420.

[15] S. Kuyucak and M. Honma, to be published.

[16] L.M. Robledo, Phys. Rev. C 46 (1992) 238.

\section{Figure Captions}

Fig. 1. Energy surface with $\pi=+1$ (solid line) and without PP (dashed line) for various values of $\eta_{3}$ ranging from 4 to 400 . The former corresponds to the critical value without PP. The energy surface with $\pi=-1$ is also shown in the top figure (dotted line) but it is too high in energy to show in the other cases.

Fig. 2. Shape-phase diagram for spherical $\rightarrow$ octupole deformation with PP (solid line) and without PP (dashed line). Deformations, obtained from the minima of the energy surfaces Eqs. (6) and (14), are plotted against $1 / \eta_{3}=N \kappa_{3} / \epsilon_{f}$. 

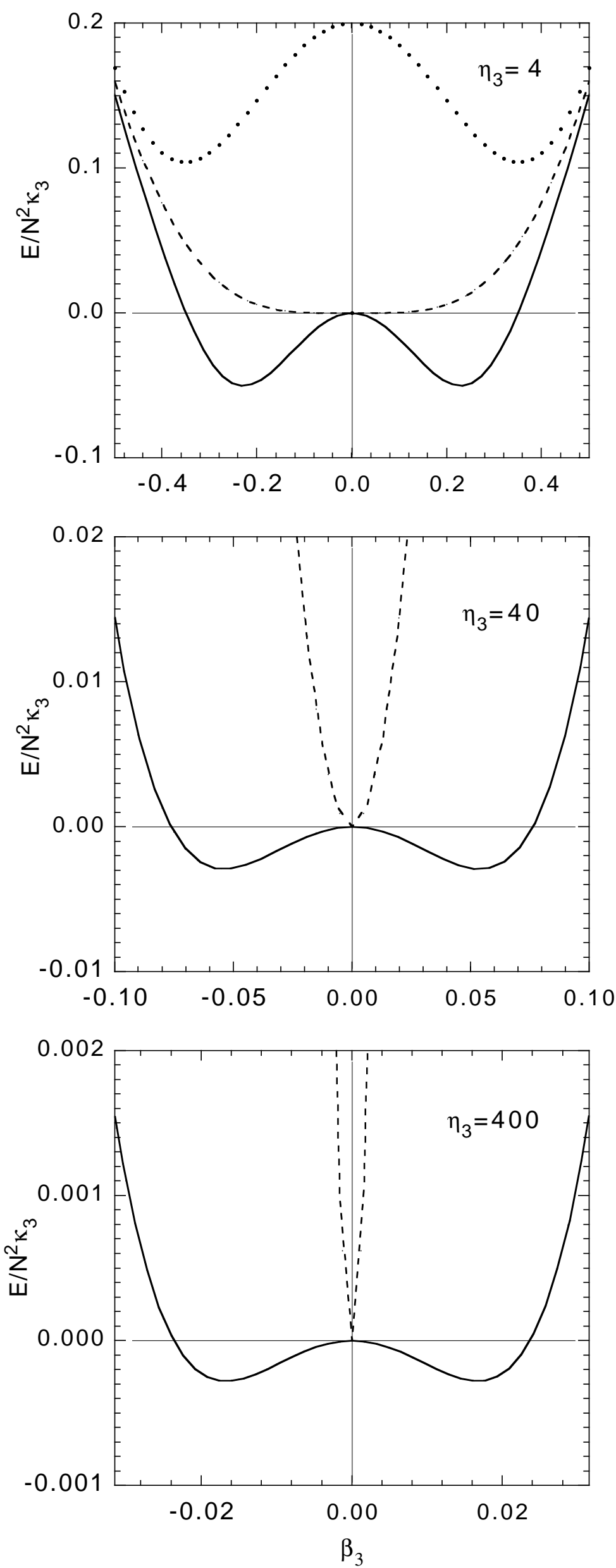


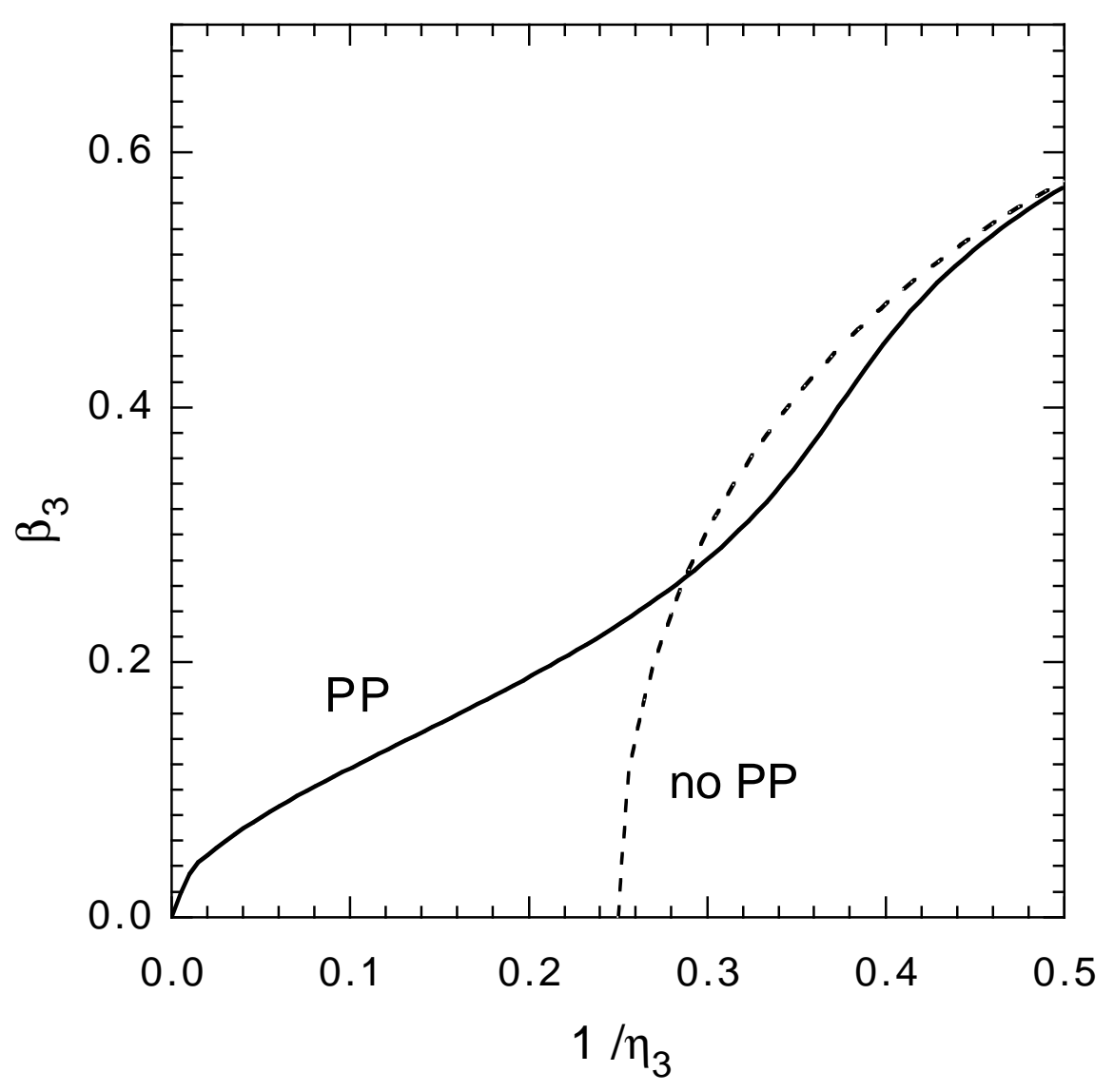

